\title{
Mycoplasma pneumoniae infection with neurologic complications
}

\author{
Alberto Spalice \\ From 71st Congress of the Italian Society of Pediatrics. Joint National Meeting SIP, SIMGePeD, Study Group \\ on Pediatric Ultrasound, SUP Study Group on Hypertension \\ Rome, Italy. 4-6 June 2015
}

Mycoplasma pneumoniae (M. pneumonia) is one of the important causes of upper and/or lower respiratory tract infections during childhood. Central nervous system (CNS) related findings and complications are most commonly seen and have been described in patients with M. pneumoniae infections $[1,2]$. Patients suffering M. pneumoniae infection may have varying degrees of neurological complications at a ratio of approximately $6-7 \%[1,2]$. Neurological manifestations include encephalitis, transverse myelitis, acute disseminated encephalomyelitis (ADEM), Guillain-Barre syndrome, and thromboembolic stroke [2]. The time period between the onset of respiratory symptoms and neurological symptoms varies 2 to 14 days [3,4]. More than $80 \%$ of patients with CNS findings have concomitant respiratory infection [5].

Central nervous system complications have been reported with Mycoplasma infection. Cerebellar syndrome, polyradiculitis, cranial nerve palsies, aseptic meningitis, meningoencephalitis, acute disseminated encephalomyelitis, coma, optic neuritis, diplopia, mental confusion and, acute psychosis secondary to encephalitis, cranial nerve palsy, brachial plexus neuropathy, ataxia, choreoathetosis, and ascending paralysis (Guillain-Barre syndrome) are neurologic complications seen with M. pneumoniae infection [1].

Neuroimaging may reveal normal findings or focal diffuse edema in cases of encephalitis or meningoencephalitis. Patchy asymmetric or diffuse signal change of gray and white matter may be seen in patients with ADEM with multifocal, asymmetric foci of high signal intensity on FLAIR and T2 weighted images. A focal infarction may be seen with $M$. pneumoniae related stroke [4].
Treatment of neurologic complications of M. pneumoniae is controversial. Treatment may be adjusted according to infection mechanism such as antibiotics, corticosteroids, intravenous immunoglobulin [3-5]. Antimicrobial treatment, especially macrolides, may be sufficient for CNS involvement associated with $M$. pneumoniae, beside the beneficial effect of treatment with steroids this treatment must be considered with direct invasion of CNS by the organism when other causative agents have been excluded. Plasma exchange has also been reported and seemed to be beneficial.

Published: 30 September 2015

\section{References}

1. Waites KB, Talkington DF: Mycoplasma pneumoniae and its role as a human pathogen. Clin Microbiol Rev 2004, 17(4):697-728.

2. Christie LJ, Honarmand S, Talkington DF, Gavali SS, Preas C, Pan CY, et al: Pediatric encephalitis: what is the role of Mycoplasma pneumoniae? Pediatrics 2007, 120(2):305-313.

3. Guleria R, Nisar N, Chawla TC, Biswas NR: Mycoplasma pneumonia and central nervous system complications: a review. J Lab Clin Med 2005, 146(2):55-63.

4. Tsiodras S, Kelesidis I, Kelisidis T, Stamboulis E, Giamarellou H: Central nervous system manifestations of Mycoplasma pneumoniae infections. J Infect 2005, 51(5):343-354.

5. Shah SS: Mycoplasma pneumonia. In Principles and Practice of Pediatric Infectious Diseases.. 3 edition. Churchill, Livingstone;Long SS, Pickering LK, Prober CG 2008:979-984.

6. Shibuya H, Osamura K, Hara K, Hisada T: Clinically mild encephalitis/ encephalopathy with a reversible splenial lesion due to Mycoplasma pneumoniae infection. Intern Med 2012, 51(12):1647-1648.

doi:10.1186/1824-7288-41-S2-A67

Cite this article as: Spalice: Mycoplasma pneumoniae infection with neurologic complications. Italian Journal of Pediatrics 2015 41(Suppl 2): A67. 